\title{
An evolutionary-weighted majority voting and support vector machines applied to contextual classification of LiDAR and imagery data fusion
}

\author{
Jorge García-Gutiérrez, Daniel Mateos-García, Mariano Garcia, \\ José C. Riquelme-Santos
}

Keywords:

Contextual classification

Data fusion

LiDAR

Machine learning

Multispectral

Remote sensing

\begin{abstract}
Data classification is a critical step to convert remotely sensed data into thematic information. Environmental researchers have recently maximized the synergy between passive sensors and LiDAR (Light Detection and Ranging) for land cover classification by means of machine learning. Although object-based paradigm is frequently used to classify high resolution imagery, it often requires a high level of expertise and time effort. Contextual classification may lead to similar results with a decrease in time and costs for non-expert users. This work shows a novel contextual classifier based on a Support Vector Machine (SVM) and an Evolutionary Majority Voting (SVM-EMV) to develop thematic maps from LiDAR and imagery data. Subsequently, the performance of SVM-EMV is compared to that achieved by a pixel-based SVM as well as to a contextual classified based on SVM and MRF. The classifiers were tested over three different areas of Spain with well differentiated environmental characteristics. Results show that SVM-EMV statistically outperforms the rest (SVM, SVM-MRF) for the three datasets obtaining a 77\%, 91\% and 92\% of global accuracy for Trabada, Huelva and Alto Tajo, respectively.
\end{abstract}

\section{Introduction}

Light Detection and Ranging (LiDAR) technology is a remote sensing laser-based technology which can determine the distance to an object based on the round-trip time of an emitted pulse. LiDAR provides not only the $x-y$ position but also the coordinate $z$ for every impact. The main applications of LiDAR have been related to extraction of digital elevation models [1], estimation of forest variables [2] and development of forest inventories [3] or fuel models [4].

Accurate and consistent thematic information derived from remotely sensed data is crucial for better management and decision making of geographic areas. Although thematic maps such as land use/land cover maps have traditionally been generated from aerial and satellite images, the appearance of new sensors such as LiDAR has led to an increasing interest in data fusion with the aim of obtaining more accurate information. In this way, LiDAR and imagery data fusion have been used to map fire risk [5] or plant communities relations [6].

Machine learning has often been used to generate thematic maps, specially supervised learning techniques such as Bayesian techniques [6,7], Support Vector Machines (SVMs) [8] or decision trees [9]. Regardless of the classification technique, the literature has shown different paradigms according to the information unit to be classified. If the information unit is a pixel, the classification can be faster but also have problems related to mixed units (pixels in the frontiers of two or more different classes which give rise to subsequent "salt and pepper" noise [10]). Moreover, high resolution images can provide a much higher number of spectrally different pixels which could contribute to an increase of the complexity of the classification step. On the other hand, we can find object-based classification which is built after an initial image segmentation. Then, the resulting limited areas (objects) can be used as information units in the classification avoiding the problems of mixed pixels and labels compound by different 
spectral responses. Unfortunately the process of building objects by segmentation is not easy and depends on the experience of the user which usually follows a trial-and-error process [11].

Contextual classification [10] can be seen as a trade-off paradigm between object and pixel orientation which, under certain conditions, can outperform object-based classification [12]. It is based on the use of the information of neighbors in addition to the information unit itself (usually a pixel) to improve final classification. It differs from ensembles such as Random Forest or Stacking (e.g. EVOR-STACK [13]) by the fact that contextual classification is usually applied in several steps (iterations) and each iteration involves a process of complete classification of the scene. On the other hand, ensembles are classification techniques that only classify the scene completely once.

Contextual classification techniques are usually grouped in preprocessing (e.g. [14]) and post-processing (e.g. [15,16]) techniques depending on the moment at which neighbor information is used in the classification process, i.e. before or after the classification, respectively. Contextual classification has proved to be suitable not only for land use/land cover mapping [13,17] but for urban classification [18].

Recently, most authors have put their efforts on the improvement of Support Vector Machines (SVM) as an example of supervised technique that has shown good results on remote sensing data [19]. Thus, researchers have developed contextual steps for the initial SVM results obtaining promising results [20]. Tarabalka et al. [17] showed one of the most recent examples of a post-processing contextual classifier applied to remote sensing. The authors proposed a technique, called SVM-MRF, for classification of hyperspectral images consisting of two steps. In the first step, a probabilistic SVM was applied to classify a hyperspectral image. In the second step, spatial contextual information was used for refining the classification results with a Markov Random Fields (MRF) regularization.

Although results obtained by SVM-MRF and other contextual techniques proved to be suitable to work with imagery, these techniques have been barely tested on LiDAR and imagery data fusion. Furthermore, evolutionary computation have already been applied to LiDAR with good results $[13,21]$ and its power to optimize processes may be an interesting way to improve contextual classification. Also, absence of statistical validation of the results can be an another important issue to extract general conclusions from previous studies in the literature.

This work presents a novel contextual classifier we called SVMEMV to develop land use/land cover maps from high resolution LiDAR and imagery data fusion. SVM-EMV is based on a classical majority voting scheme but taking advantage of a new evolutionary adaptation which lets SVM-EMV improve classical contextual classification results. Subsequently, we compare the performance of SVM-EMV with those of SVM and SVM-MRF after testing on data from three sites in the Iberian peninsula, previously used in relevant publications. Finally, the results are statistically validated and discussed.

This paper is organized in the following manner for a better understanding: Section 2 provides a description of the methodology and the main features of the data used in this work. Section 3 shows and analyzes the results achieved. Finally, Section 4 presents the main conclusions of the study and suggests future lines of research.

\section{Materials and methods}

\subsection{Data sites description}

This study was based on data from three different areas in the Iberian Peninsula (see Fig. 1). The first study site was located in

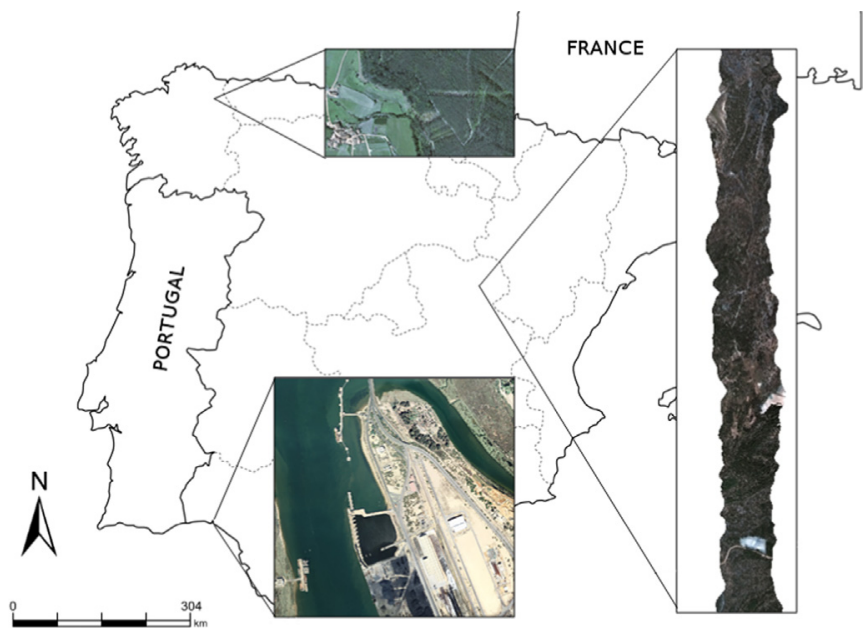

Fig. 1. Study areas: Trabada in the north, Huelva in the south, and Alto Tajo in the center of Spain.

Trabada (hereafter site A) and covered a rectangle of about $0.6 \times 0.45 \mathrm{~km}^{2}$ in the municipality of Vilapena (Galicia, NW Spain, UTM29; 644637E 4808199N). The area was dominated by eucalyptus stands, with low intensity silvicultural treatments and the presence of tall shrubs. In addition, three other labels were taken into account: roads, crops and buildings.

The data for the second study site (hereafter site B) were collected from a coastal area of the province of Huelva (SW Spain, UTM30; 150960E $4124465 \mathrm{~N}$ ). The study site covered an area of $1.5 \times 1.5 \mathrm{~km}^{2}$ in the mouth of the Tinto and Odiel rivers. This site presented a mix of land uses and cover. Eight labels were selected for the final classification: water, marshland, roads and railways, low vegetation (pastures), middle vegetation (bushes), tall vegetation (mainly eucalyptus), buildings (industrial areas, port facilities) and landfills.

LiDAR data and aerial orthophotography (with resolution $1 \mathrm{~m}$ for site A and $0.5 \mathrm{~m}$ for site B and only visible spectrum bands: red, green and blue) were provided for both data sites which had already been presented and used in previous works after preprocessing (see Garcia-Gutierrez et al. [9] for a deeper explanation about the preprocessing techniques applied)

The last study site (hereafter site C) was located in the Natural Park of the Alto Tajo in Guadalajara (central Spain, UTM30; $563385 \mathrm{E} 4533140 \mathrm{~N}$ ). This study covered an area of approximately $9 \times 0.3 \mathrm{~km}^{2}$. The target labels studied comprised: roads, bare soil, pine, poplar, two types of pasture, two types of old oak, young oak and shrub.

For this site, Airborne Thematic Mapper (ATM) data (multispectral images) with a resolution of $2 \mathrm{~m}$ along with LiDAR were collected. From the ATM data, spectral bands covering the visible, near infrared and shortwave infrared regions of the electromagnetic spectrum were used. After studying the density of the LiDAR data (1-2 pulses $/ \mathrm{m}^{2}$ ), the image was resampled to $6 \mathrm{~m}$ using the mean value of the pixels. Then four spectral indices were derived to enhance the spectral response of vegetation and reduce the effect of the ground on the signal. Subsequently, spectral indices and multispectral layers were stacked into an image to be used beside the LiDAR information. Further information on the processing of these data can be found in Garcia et al. [22].

\subsection{Feature set extraction}

A vector of features (numerical attributes) was attached to each cell (pixel) in the matrix that would give rise to each thematic layer. Vectors were calculated as a stack of image bands (red, green 
Table 1

Features obtained from the ATM image (bands and spectral indexes).

\begin{tabular}{ll}
\hline Band & Spectral range $(\mathrm{nm})$ \\
\hline B1 & $420-450$ \\
B2 & $450-520$ \\
B3 & $520-600$ \\
B4 & $600-620$ \\
B5 & $630-690$ \\
B6 & $690-750$ \\
B7 & $760-900$ \\
B8 & $910-1050$ \\
B9 & $1550-1750$ \\
B10 & $2080-2350$ \\
Spectral index & Definition \\
\hline NDVI & $\left(B_{7}-B_{5}\right) /\left(B_{7}+B_{5}\right)$ \\
SAVI & $\left(B_{7}-B_{5}\right) \times 1.5 /\left(B_{7}+B_{5}\right)$ \\
NDII $_{1}$ & $\left(B_{7}-B_{9}\right) /\left(B_{7}+B_{9}\right)$ \\
NDII $_{2}$ & $\left(B_{7}-B_{1} 0\right) /\left(B_{7}+B_{1} 0\right)$ \\
\hline
\end{tabular}

Table 2

LiDAR-based textures calculated for both LiDAR intensities and normalized heights.

\begin{tabular}{ll}
\hline Symbol & Description \\
\hline MAX & Maximum \\
MIN & Minimum \\
RANG & Range \\
STD & Standard deviation \\
VAR & Variance \\
MEAN & Mean \\
KURT & Kurtosis \\
CV & Coefficient of variation \\
SKEW & Skewness \\
MED & Median \\
ENT & Entropy \\
WRFR & Weighted rank fill ratio \\
\hline
\end{tabular}

and blue bands for orthophotos; see Table 1 for a description of the multispectral bands), spectral indexes calculated from the available bands (multispectral only) and LiDAR-derived statistics.

Before extracting the LiDAR statistics, a resolution had to be set up. It was selected according to the resolution of the images and the density of the LiDAR pulses. The resolution was set at $1 \mathrm{~m}^{2}$ for site A, $4 \mathrm{~m}^{2}$ for site B and $6 \mathrm{~m}^{2}$ for site C. Table 2 shows the LiDAR statistics. All of them were calculated from both LiDAR intensity and normalized-height distributions which gave rise to a set of 24 new attributes. After the stacking of the LiDAR and image features, each pixel finally included 27 numeric attributes for sites A and B and 38 for site $\mathrm{C}$.

\subsection{SVM classification}

Two subsets of pixels were selected to be the training and test sets for each site. After the extraction of the two sets of pixels, three types of filters (from Weka [23] with default parameters) were applied. First, the missing values were replaced by the corresponding mean value. Second, the data were normalized. Finally, to avoid problems with dimensionality, a feature selection based on correlations was applied (CFS filter). Using the filtered training set, a SVM with a Gaussian kernel was developed. The parameters of the SVM were optimized by an evolutionary algorithm according to the guidelines given in the literature [24].

\subsection{SVM-EMV}

Our classification method (called SVM-EMV) consisted of a SVM in a first level of classification and a post-processing step based on an Evolutionary Majority Voting in a second one. Thus, once the initial classification was done by the SVM referred in the previous subsection, the pixel instances were transformed into a new type of data, i.e. the set of Euclidean distances from each pixel to its neighbors. A vote system was then applied to reclassify every pixel. The vote system selected the most common label among the neighbors taking into account that each neighbor vote was weighed according to the distance to the pixel to be reclassified. This process is applied to every pixel in several iterations ( 5 in our case since this number assures convergency for MRF [10]). Following Tarabalka et al. [17] neighbor pixels were selected using a 8pixel adjacency criterion.

The optimal weights for each position in the vector of distances were obtained by an evolutionary procedure which tried to optimized the reclassification of the training instances. To determine an evolutionary algorithm, it is necessary to define an individual encoding, genetic operators, a fitness function and a rule to carry out the generational replacement. These aspects are described in the following subsections.

\subsubsection{Individual encoding}

In this work, an individual was a set of weights or relative vote coefficients and was represented by a vector with a real value for each neighbor and also for the pixel to be reclassified. Therefore, each vector comprised 9 elements. We included the pixel to be classified as another neighbor with distance 0.0 to represent the probability of having reached an optimal classification by a previous step (initially by SVM).

Each element of the individual was a real value whose initial lower and upper bounds were customizable. In our case, the chosen range was $[0,1]$ but it was possible to reach values above one through the crossover and mutation operators. The initial population consisted of $N$ (in our case, 100 individuals) real-valued vectors with 9 random values between 0 and 1 . The identity individual (every weight with a value of 1 ) was always included in the initial population.

\subsubsection{Genetic operators}

The crossover operation between two individuals was carried out uniformly (random selection of the $i$-th coefficient from the two $i$-th values in the parents). The mutation operator was designed to randomly increase or decrease the value of a gene $w$ in a value $\delta * w$ with probability $p$. Initially, $\delta \in[0,1]$, but for a better fit, every $g$ generations, the upper bound was reduced by $g / G$, where $G$ is the total number of generations. By a trial-and-error procedure, we selected $G=100$ and $g=10$; therefore, during the first ten generations, the upper bound for $\delta$ was 1 , in the following ten it was 0.9 , in the next ten 0.8 , etc.

\subsubsection{Fitness function}

Fig. 2 shows the Evaluate procedure used in the fitness function of the evolutionary method. Each individual (vector of weights) was represented by the $W$ input parameter (line 1 ). Every training instance comprised the eight labels of the neighbors of the pixel to be classified and the one of the pixel itself at that moment, increasingly sorted by their distances to the latter. The parameter lab stands for the real label of the pixel to be classified according to the training set.

The fitness value for an individual is the accumulated Evaluate return for each instance of the training set. Thus, for each $i$-th label in a given instance the relative $i$-th vote from the vector $W$ was 


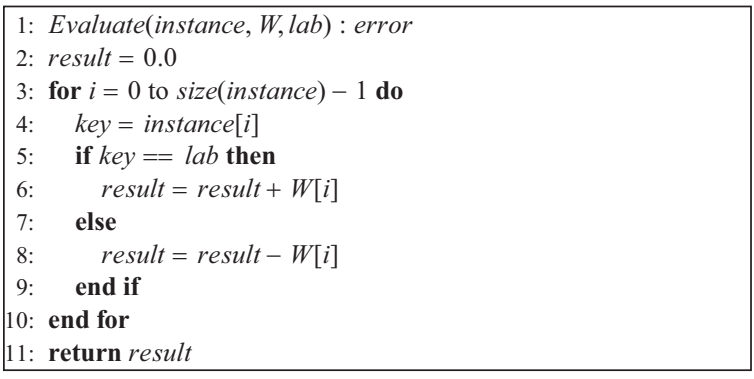

Fig. 2. Fitness function.

Table 3

Number of training and test instances for each site.

\begin{tabular}{lll}
\hline Site & Training & Test \\
\hline A & 1664 & 306 \\
B & 4013 & 616 \\
C & 1209 & 532 \\
\hline
\end{tabular}

accumulated by Evaluate if the label coincides with the reference one $(l a b)$. Otherwise, the vote is subtracted as penalization. Finally, the method Evaluate returned the accumulated value. The final fitness of an individual $W$ may therefore be defined as the sum of the well-classified weights minus the misclassified ones of the neighbors of the pixels in the training set.

\subsubsection{Generational replacement}

For the replacement of individuals from one generation to the next, we chose an elitist design where the best individual went to the next generation without being affected by the mutation operator and the rest were the result of the application of the genetic operators. To select the individuals that would be involved in the crossover operation we used a tournament method.

\subsection{Statistical analysis}

Classification results were obtained for SVM, SVM-MRF and SVM-EMV and a statistical comparison was established afterwards. The statistical analysis was used to check the significance in the differences among the studied methods in terms of the global accuracy. To this end, we applied a Cochran's $Q$ test which is an extension of the McNemar's test for multiple classifiers [25].

\section{Results}

Table 3 shows the number of training and test samples used in this work for each site. Furthermore, Table 4 shows the final set of features for each site after the analysis of separability by the remote sensing experts who provided the data and the later preprocessing and feature selection phase on the training data. Finally, we provide the selected parameters used to optimize the SVM performance in Table 5.

We evaluated the performance of SVM, SVM-MRF and SVM-EMV on the test set referred in Table 3. Due to the random nature of the evolutionary algorithms, the EMV results were those obtained by the run which obtained the median result in 5 repetitions.

We provide a summary of the confusion matrices after a hold-out validation (common validation in remote sensing) in Tables 6-8. For every site, SVM-EMV obtained the best results. Tables show global accuracy, Kappa index and F-measure. F-measure is a well-known statistic in machine learning to evaluate commission and omission errors which enables a comparison of the per-class results of the three
Table 4

Attributes selected after feature selection phase.

\begin{tabular}{ll}
\hline Site & Features \\
\hline A & R, B, HMEAN, HMIN, HMAX, HMED, \\
& HENT, IMEAN, IMIN, IRANGE, ICV. \\
B & R, G, B, HMEAN, HVAR, HSTDV, \\
& HMIN, HMAX, HSKEW, \\
& HMED, HCV, HENT, IMIN, \\
& IKUT, IMED, IENT. \\
C & B2, B6, B7, B8, \\
& B10, SAVI, NDII ${ }_{1}$, \\
& HMAX, HMED, HSTDV, HRANGE. \\
\hline
\end{tabular}

Table 5

Parameters selected to optimize each SVM.

\begin{tabular}{lll}
\hline Site & C & $\gamma$ \\
\hline A & 205.33 & 9.1 \\
B & 823.16 & 0.01 \\
C & 183.66 & 0.2 \\
\hline
\end{tabular}

Table 6

Summary of the hold-out test on dataset A. F-measure obtained for every label and global statistics (accuracy and kappa index). In bold, best values.

\begin{tabular}{llll}
\hline Labels & SVM & SVM-MRF & SVM-EMV \\
\hline Dataset A confusion matrix & & & \\
Roads & 0.50 & 0.56 & $\mathbf{0 . 6 1}$ \\
Crops & 0.73 & 0.76 & $\mathbf{0 . 7 8}$ \\
Eucalyptus & 0.78 & 0.83 & $\mathbf{0 . 8 5}$ \\
Buildings & 0.69 & 0.74 & $\mathbf{0 . 7 9}$ \\
Global accuracy & 0.69 & 0.74 & $\mathbf{0 . 7 7}$ \\
Kappa & 0.57 & 0.63 & $\mathbf{0 . 6 7}$ \\
\hline
\end{tabular}

Table 7

Summary of the hold-out test on dataset B. F-measure obtained for every label and global statistics (accuracy and kappa index). In bold, best values.

\begin{tabular}{llll}
\hline Labels & SVM & SVM-MRF & SVM-EMV \\
\hline Dataset B confusion matrix & & & \\
Water & $\mathbf{1 . 0}$ & $\mathbf{1 . 0}$ & $\mathbf{1 . 0}$ \\
Marshland & 0.93 & 0.95 & $\mathbf{0 . 9 6}$ \\
Roads & 0.83 & 0.83 & $\mathbf{0 . 8 4}$ \\
Low vegetation & 0.87 & $\mathbf{0 . 9 0}$ & $\mathbf{0 . 8 8}$ \\
Middle vegetation & 0.75 & 0.86 & $\mathbf{0 . 8 8}$ \\
High vegetation & 0.91 & 0.95 & $\mathbf{0 . 9 9}$ \\
Buildings & 0.80 & 0.81 & $\mathbf{0 . 8 3}$ \\
Dumps & 0.90 & $\mathbf{0 . 9 7}$ & $\mathbf{0 . 9 7}$ \\
Global accuracy & 0.90 & 0.91 & $\mathbf{0 . 9 2}$ \\
Kappa & 0.88 & 0.89 & $\mathbf{0 . 9 0}$ \\
\hline
\end{tabular}

algorithms. As can be seen, SVM-EMV obtained the best results again. Only in sites B and C, the two contextual algorithms interchanged positions in four cases though SVM-EMV mostly reached the best result per label.

Finally, to validate our study, a Cochran's $Q$ test was applied to the results obtained by each algorithm in sites $\mathrm{A}, \mathrm{B}$ and $\mathrm{C}$. The test provided a $p$-value lower than $0.001,0.01$ and 0.001 , respectively. The null hypothesis for Cochran's $Q$ test was that the algorithms did not behave significantly different. The $p$-values thus rejected the null hypothesis for each site with a level of significance of $\alpha=0.05$.

Although a hold-out validation is the most extended technique to test remote sensing classification, even with smaller datasets 
than those used in this work [17], its use can potentially bias the conclusions given the small size of the datasets used. For this reason, we established a second level of validation based on a 10fold cross-validation (10FCV) after fusing both training and test sets for each site. Results can be seen in Table 9. For every site, SVM-EMV obtained the best results in terms of averaged global accuracy and lower standard deviation which involves a higher level of stability across the 10 folds.

For the concrete case of SVM-EMV, we provide some learning curves (Fig. 3 ) showing the evolutionary process. Also Fig. 4 shows the evolution during the 5 iterations for both contextual techniques (SVM-MRF and SVM-EMV) in terms of relative improvement

Table 8

Summary of the hold-out test on dataset C. F-measure obtained for every label and global statistics (accuracy and kappa index). In bold, best values.

\begin{tabular}{llll}
\hline Labels & SVM & SVM-MRF & SVM-EMV \\
\hline Dataset C confusion matrix & & & \\
Young oak & 0.74 & 0.86 & $\mathbf{0 . 8 8}$ \\
Bare soil & 0.98 & $\mathbf{0 . 9 9}$ & 0.96 \\
Roads & 0.77 & 0.88 & $\mathbf{0 . 9 1}$ \\
Bushes & 0.75 & 0.85 & $\mathbf{0 . 8 6}$ \\
Poplar & 0.84 & $\mathbf{1 . 0}$ & $\mathbf{1 . 0}$ \\
Old holm oak 1 & 0.77 & $\mathbf{0 . 9 2}$ & $\mathbf{0 . 9 2}$ \\
Old holm oak 2 & 0.90 & $\mathbf{0 . 9 7}$ & 0.96 \\
Pasture 1 & 0.77 & 0.84 & $\mathbf{0 . 8 5}$ \\
Pasture 2 & 0.97 & $\mathbf{0 . 9 8}$ & $\mathbf{0 . 9 8}$ \\
Pine & 0.92 & 0.96 & $\mathbf{0 . 9 8}$ \\
Global accuracy & 0.84 & 0.91 & $\mathbf{0 . 9 2}$ \\
Kappa & 0.81 & 0.90 & $\mathbf{0 . 9 1}$ \\
\hline
\end{tabular}

Table 9

Mean and standard deviation obtained by each technique for each dataset on a 10fold cross-validation. In bold, the best values.

\begin{tabular}{|c|c|c|c|c|c|c|}
\hline \multirow[t]{2}{*}{ Method } & \multicolumn{2}{|l|}{ Site A } & \multicolumn{2}{|l|}{ Site B } & \multicolumn{2}{|l|}{ Site C } \\
\hline & Mean & Stdv & Mean & Stdv & Mean & Stdv \\
\hline SVM & 91.97 & 1.49 & 91.75 & 8.46 & 83.13 & 2.24 \\
\hline SVM-MRF & 94.35 & 1.61 & 93.26 & 8.99 & 91.24 & 2.31 \\
\hline SVM-EMV & 94.88 & 1.37 & 93.49 & 7.29 & 91.63 & 1.99 \\
\hline
\end{tabular}

Fitness vs. Iterations (Site A)

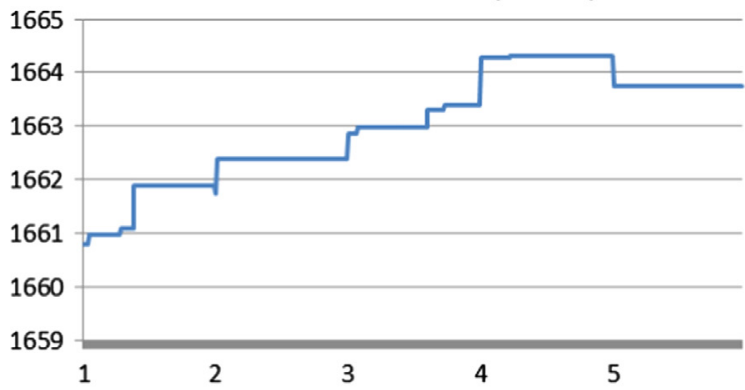

regarding SVM. Finally, the results obtained by SVM-EMV can be seen in Fig. 5 where a sample of the maps developed is shown.

After analyzing the results obtained, we should outline some important findings. First, feature selection phase draws the importance of LiDAR when the spectral information is limited in the images. Thus, we find that for sites A and B, where only RGB was available, the process selected intensity-derived features to include near-infrared information in the classification process. For site C, the intensity was ignored because ATM had already provided that information. Furthermore, every selection included statistics related to heights which reinforce the LiDAR role in the final classification.

Regarding the parameters selected for the SVM, note that the gamma value for the site A was very high in comparison with the rest of sites. This parameter intuitively indicates how far the influence of a single training example reaches and, beside a high penalization value for each error $(\mathrm{C})$, resulted in a possible overfitting situation. This fact is closely linked to the discrepancies found between the hold-out validation and the 10FCV. The 10FCV results show a much better performance which can be due to a validation technique less potentially biased. All in all, both validations showed SVM-EMV as the best technique regardless the concrete results.

The results of SVM-EMV showed good overall results for the fusion of LiDAR and imagery. They were better for the more complex datasets with more classes than for site A where there were only 4 labels but this may be caused by an insufficient number of examples for the classification of the complete scene and or overfitting problems. Regarding the results for the other sites (B and C), EMV obtained good results comparable with those from object-based approaches. Even when it is possible to find higher accuracies in the literature [26] for similar classification by object orientation, development time is another key factor to take care of. In our case, the classification was done in less than $1 \mathrm{~h}$ for every site in a personal computer (four I7 processors at $2.7 \mathrm{GHz}$ and 8 GB RAM). Since evolutionary computation was responsible for the parameterization, SVM-EMV showed its potential as an industrial tool for faster thematic map generation (object-oriented maps can take even months of work [27]).

Analyzing the results from site $\mathrm{C}$, we found that EMV obtained similar accuracies than those obtained in the original work (e.g. original global accuracy around 91.4\%). However those similar results had been obtained after grouping spectral classes into informational classes, i.e. one type of grass, one of holm oak, etc. This step had increased the

Fitness vs. Iterations (Site C)

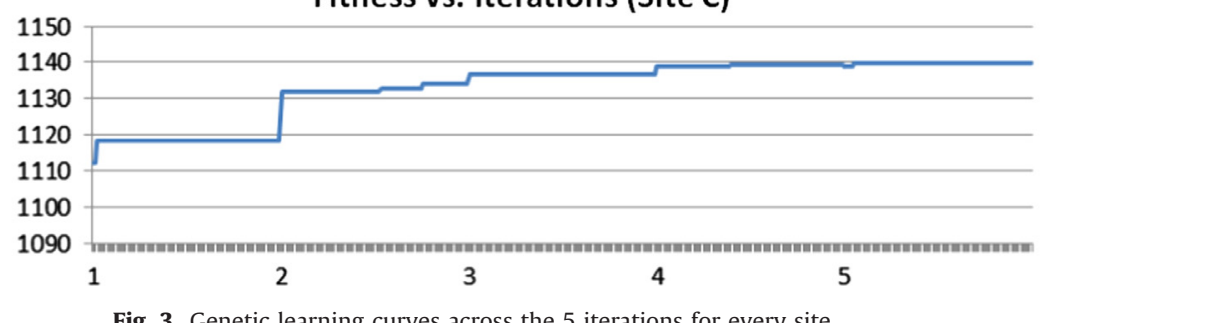

Fig. 3. Genetic learning curves across the 5 iterations for every site.

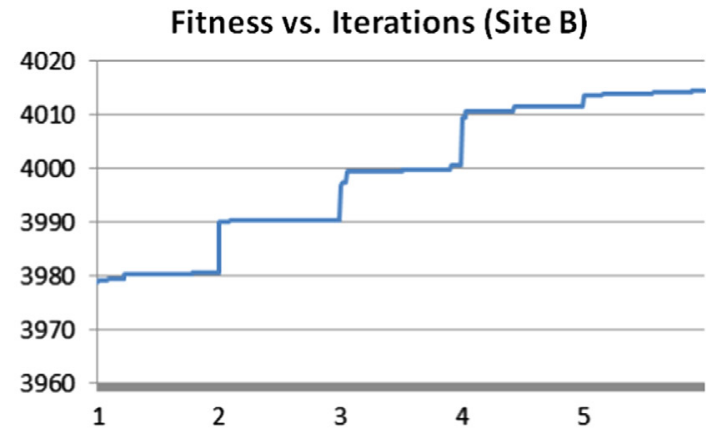




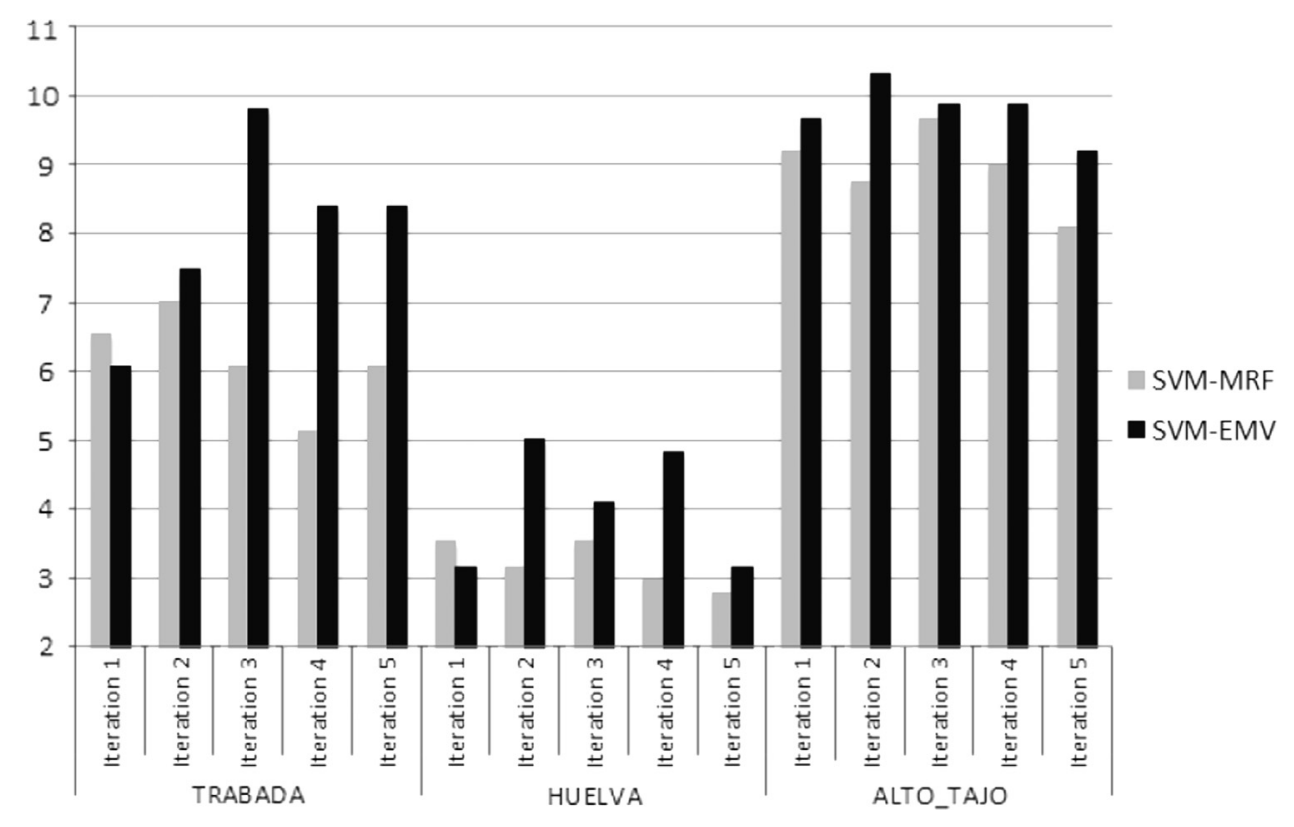

Fig. 4. Percentage of relative improvement from SVM results.

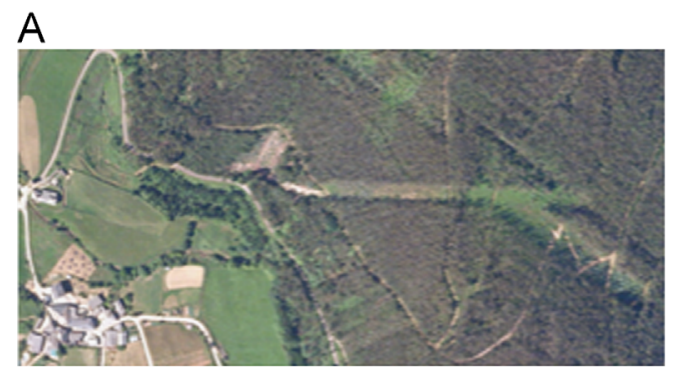

\section{B}
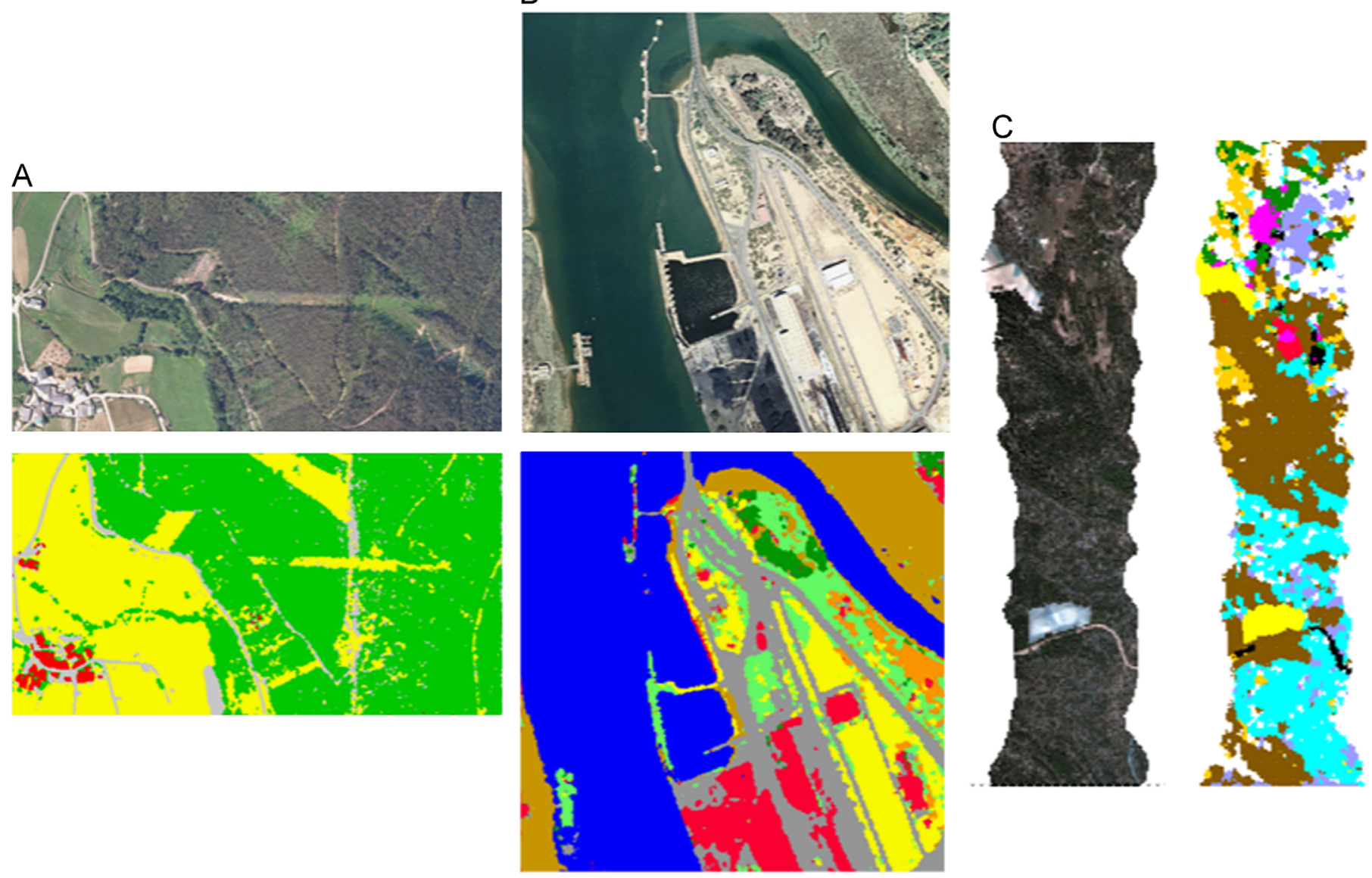

Fig. 5. Samples of the final classification developed by SVM-EMV, from left to right sites A, B and C.

global accuracy and therefore the fact that EMV obtained an even greater accuracy with 10 classes (92.2\%) instead of 6 classes should be taken into account.
Another interesting point is how SVM-EMV evolves over time. In Fig. 3, we can see how the fitness improved over time except when EMV passed from one iteration to the next. As the contextual 
algorithm completely changes (classifies) the scene, the fitness of the next evolutionary step may be affected, specially when the elitism does not propagate to the next execution (something to be tested in future work). Furthermore, it is assumed that contextual algorithms (at least MLR) tend to stabilize at a number of iterations though, it can be seen that supposed stabilization has no effect on the accuracy obtained (see Fig. 4) because this type of classification tends to eliminate smallest details. For the contextual algorithm completely changes the scene, the accuracy of the next iteration may be affected giving rise to oscillations across the iterations. Anyway, SVM-EMV obtained better results regardless the iteration selected apart from the first one though future work should also pay special attention to the selection of its stopping criterion.

Even taking into account the previous problems, our approach obtained the best global and per-label results (with some minor exceptions in sites B and C) and it should therefore be taken into account as a valid technique to improve the general results obtained by SVMs with similar or better accuracies than the best contextual techniques. The global accuracies reached were also comparable with the results of the classification with objectoriented techniques (except for site A) but with a considerable decrease in the time of development a1nd minimal previous knowledge required about the classifier selected (SVM).

\section{Conclusions}

This research compared the performance of SVMs and other two contextual classifiers (SVM-MRF and a novel classifier called SVMEMV) to categorize land cover on LiDAR and optical data fusion in three areas of Spain (Trabada, Huelva and Alto Tajo). The results showed that SVM-EMV outperformed the rest and obtained accuracies over $91 \%$ for the Huelva and Alto Tajo sites. The statistical tests confirmed the better performance of the SVM-EMV classifier compared with the other classifiers tested. Although there were little difference between the contextual classifiers, they were still statistically significant.

Future work should compare pixel-oriented, contextual and objectoriented approaches [28]. Contextual classifiers and object-oriented approaches could solve some pixel-inherent problems, including saltand-pepper noise using pixel neighborhood or objects as instances to classify, respectively. The high suitability level shown by contextual classifiers in this study should be confirmed after a more extensive comparison against those different paradigms. New test sites could reinforce the results obtained in this study and therefore confirm SVMEMV as an adequate technique for multisource classification. Finally, imbalance in datasets is a problem not covered in this work which can seriously affect the final per-label results. A further analysis of the imbalance problem and its effects on our approach will also be carried out in future work.

\section{Acknowledgements}

Data from the area of Alto Tajo were acquired by the UK Natural Environment Research Council: Airborne Remote Sensing Facility 2006 Mediterranean Campaign, Grant WM06-04 awarded to Prof. Mark Danson, University of Salford. Data from Huelva were acquired by the Regional Ministry of Andalusia and formed part of the project "Detailed Mapping and Web Dissemination of Demographic, Tourist and Environmental Data for Vulnerability Assessment Linked to Beach Erosion Along the Andalusian Coast (CSO2010-15807)". The data used from Trabada were funded by the Galician Government (Xunta de Galicia) and Land Laboratory (Prof. David Miranda and Prof. Rafael Crecente, University of
Santiago de Compostela). Finally, the authors would like to thank Spanish Ministry of Science and Technology and Regional Ministry of Andalusia for the support under projects TIN2011-28956-C02 and P11-TIC-7528, respectively.

\section{References}

[1] A. Brzank, C. Heipke, J. Goepfert, U. Soergel, Aspects of generating precise digital terrain models in the Wadden sea from LiDAR, water classification and structure line extraction, ISPRS J. Photogramm. Remote Sens. 63 (2008) $510-528$.

[2] E. González-Ferreiro, D. Miranda, L. Barreiro-Fernandez, S. Bujan, J. GarciaGutierrez, U. Dieguez-Aranda, Modelling stand biomass fractions in Galician eucalyptus globulus plantations by use of different LiDAR pulse densities, For. Syst. 22 (2013) 10-23.

[3] J.E. Anderson, L.C. Plourde, M.E. Martin, B.H. Braswell, M.-L. Smith, R.O. Dubayah, M.A. Hofton, J.B. Blair, Integrating waveform LiDAR with hyperspectral imagery for inventory of a northern temperate forest, Remote Sens. Environ. 112 (2008) 1856-1870.

[4] M. Garcia, D. Riano, E. Chuvieco, F.M. Danson, Estimating biomass carbon stocks for a Mediterranean forest in central Spain using LiDAR height and intensity data, Remote Sens. Environ. 114 (2010) 816-830.

[5] B. Koetz, F. Morsdorf, S. van der Linden, T. Curt, B. Allgower, Multi-source land cover classification for forest fire management based on imaging spectrometry and LiDAR data, For. Ecol. Manag. 256 (2008) 263-271.

[6] J. Verrelst, G.W. Geerling, K.V. Sykora, J.G. Clevers, Mapping of aggregated floodplain plant communities using image fusion of CASI and LiDAR data, Int. J. Appl. Earth Obs. Geoinf. 11 (2009) 83-94.

[7] K. Anderson, J. Bennie, E. Milton, P. Hughes, R. Lindsay, R. Meades, Combining LiDAR and IKONOS data for ecohydrological classification of an ombrotrophic peatland, J. Environ. Qual. 39 (2010) 260-273.

[8] J. Garciagutierrez, D. Mateos-Garcia, J.C. Riquelme, A SVM and k-NN restricted stacking to improve land use and land cover classification, Lecture Notes in Computer Science, vol. 6077, Springer, Berlin, Heidelberg (2010) 493-500.

[9] J. Garcia-Gutierrez, L. Gonçalves-Seco, J.C. Riquelme-Santos, Automatic environmental quality assessment for mixed-land zones using LiDAR and intelligent techniques, Expert Syst. Appl. 38 (2011) 6805-6813.

[10] F.J. Cortijo, N.P.D.L. Blanca, Improving classical contextual classifications, Int. J. Remote Sens. 19 (1998) 1591-1613.

[11] R. Hamilton, K.A. Megown, T.C. Mellin, I. Fox, Guide to Automated Stand Delineation Using Image Segmentation, U.S. Department of Agriculture, Forest Service, Remote Sensing Applications Center, Salt Lake City, Utah, 2007.

[12] G. Thoonen, K. Hufkens, J.V. Borre, T. Spanhove, P. Scheunders, Accuracy assessment of contextual classification results for vegetation mapping, Int. J. Appl. Earth Obs. Geoinf. 15 (2012) 7-15.

[13] J. Garcia-Gutierrez, D. Mateos-Garcia, J.C. Riquelme-Santos, EVOR-STACK: a label-dependent evolutive stacking on remote sensing data fusion, Neurocomputing 75 (2012) 115-122.

[14] T. Hermosilla, L. Ruiz, J. Recio, M. Cambra-Lopez, Assessing contextual descriptive features for plot-based classification of urban areas, Landsc. Urban Plan. 106 (2012) 124-137.

[15] L. Luo, G. Mountrakis, Converting local spectral and spatial information from a priori classifiers into contextual knowledge for impervious surface classification, ISPRS J. Photogramm. Remote Sens. 66 (2011) 579-587.

[16] A.L. Levada, N.D. Mascarenhas, A. Tannús, A novel MAP-MRF approach for multispectral image contextual classification using combination of suboptimal iterative algorithms, Pattern Recognit. Lett. 31 (2010) 1795-1808.

[17] Y. Tarabalka, M. Fauvel, J. Chanussot, J. Benediktsson, SVM- and MRF-based method for accurate classification of hyperspectral images, IEEE Geosci. Remote Sens, Lett. 7 (2010) 736-740.

[18] J. Niemeyer, F. Rottensteiner, U. Soergel, Contextual classification of LiDAR data and building object detection in urban areas, ISPRS J. Photogramm. Remote Sens. 87 (2014) 152-165.

[19] G. Mountrakis, J. Im, C. Ogole, Support vector machines in remote sensing: a review, ISPRS J. Photogramm. Remote Sens. 66 (2011) 247-259.

[20] R.G. Negri, L.V. Dutra, S.J.S. SantAnna, An innovative support vector machine based method for contextual image classification, ISPRS J. Photogramm. Remote Sens. 87 (2014) 241-248.

[21] J. Garcia-Gutierrez, E. Gonzalez-Ferreiro, J.C. Riquelme-Santos, D. Miranda, U. Dieguez-Aranda, R.M. Navarro-Cerrillo, Evolutionary feature selection to estimate forest stand variables using LiDAR, Int. J. Appl. Earth Obs. Geoinf. 26 (2014) 119-131.

[22] M. Garcia, D. Riano, E. Chuvieco, J. Salas, F.M. Danson, Multispectral and LiDAR data fusion for fuel type mapping using support vector machine and decision rules, Remote Sens. Environ. 115 (2011) 1369-1379.

[23] M. Hall, E. Frank, G. Holmes, B. Pfahringer, P. Reutemann, I.H. Witten, The WEKA data mining software: an update, SIGKDD Explor. 11 (2009).

[24] C.-L. Huang, C.-J. Wang, A GA-based feature selection and parameters optimization for support vector machines, Expert Syst. Appl. 31 (2006) 231-240.

[25] D. Sheskin, Handbook of Parametric and Nonparametric Statistical Procedures, third ed., Chapman \& Hall, Boca Raton, 2004. 
[26] A. Antonarakis, K. Richards, J. Brasington, Object-based land cover classification using airborne LiDAR, Remote Sens, Environ. 112 (2008) 2988-2998.

[27] L. Barreiro Fernández, J. García Gutiérrez, E. González Ferreiro, S. Buján Seoane, L. Gonçalves Seco, D. Miranda Barrós, Land Cover Classification of Forest Areas Using LiDAR and Spectral Data, 2010.

[28] D. Lu, Q. Weng, A survey of image classification methods and techniques for improving classification performance, Int. J. Remote Sens. 28 (2007) 823-870. 